\title{
TENDÊNCIAS DA FUNÇÃO DO ENFERMEIRO AUDITOR NO MERCADO EM SAÚDE
}

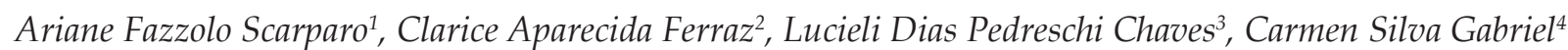

${ }^{1}$ Doutoranda do Programa de Enfermagem Fundamental da Escola de Enfermagem de Ribeirão Preto (EERP) da Universidade de São Paulo (USP). Enfermeira do Hospital São Paulo de Ribeirão Preto-SP. São Paulo, Brasil. E-mail: pscarparo@ig.com.br 2 Doutora em Enfermagem. Professor Associado da EERP/USP. São Paulo, Brasil. E-mail: erraz@eerp.usp.br

${ }^{3}$ Doutora em Enfermagem Fundamental. Professor Doutor da EERP/USP. São Paulo, Brasil. E-mail: dpchaves@eerp.usp.br

${ }^{4}$ Doutora em Enfermagem em Saúde Pública. Professor Doutor da EERP/USP. São Paulo, Brasil. E-mail: cgabriel@eerp.usp.br

RESUMO: Este estudo objetivou identificar tendências atuais e futuras (próximos cinco anos) da função do enfermeiro auditor no mercado de trabalho. Metodologicamente a investigação foi estruturada utilizando-se a técnica Delphi, por meio de duas rodadas, com a participação final de 34 enfermeiros de instituições de saúde e ensino brasileiras. Os dados foram tratados mediante estatística descritiva. Os resultados evidenciam que na atualidade, está focada na dimensão burocrática, de cunho contábil e financeiro, contemplando exigências das instituições de saúde. Quando essa tendência é reportada para o futuro, observa-se uma projeção de mudança incorporando a avaliação da qualidade da assistência. Quanto aos métodos, a situação atual, é de proceder segundo a forma retrospectiva, do tipo interna, contemplando a coleta e análise dos pagamentos de contas. À medida que a auditoria de enfermagem incorporar à sua prática a avaliação da qualidade, conforme tendência futura, os métodos agregarão diferentes maneiras para sustentar a nova realidade.

DESCRITORES: Auditoria de enfermagem. Economia. Qualidade da assistência à saúde.

\section{TENDENCIES OF THE ROLE OF THE AUDITOR NURSE IN THE HEALTH CARE MARKET}

\begin{abstract}
This study aimed to identify current and future (over the next five years) tendencies of the role of the auditor nurse in the labor market. The research was methodologically structured using the Delphi technique, through two rounds, with a total participation of 34 nurses from Brazilian health care and teaching institutions. The data was analyzed using descriptive statistics. The results provide evidence that nursing auditing is currently focused on bureaucratic aspects such as accounting and finance, thus meeting the demands of health care institutions. Regarding the future, a projection for change is reported in order to incorporate health care quality assessment. At present, professionals act according to retrospective internal methods, dealing with the stages of collecting and analyzing the payment of bills. As nursing auditing incorporates quality assessment into its practices, as is the projected tendency for the next five years, the methods used will add different means to sustain the new reality.
\end{abstract}

DESCRIPTORS: Nursing audit. Economics. Quality of health care.

\section{TENDENCIAS DE LA FUNCIÓN DEL ENFERMERO AUDITOR EN EL SECTOR DE SALUD}

RESUMEN: Este estudio tuvo como objetivo identificar las tendencias actuales y futuras (próximos cinco años) de la función del enfermero auditor en el sector de salud. La investigación fue estructurada utilizando la técnica Delphi, a través de dos rondas, con la participación final de 34 enfermeras de instituciones de salud y educación en Brasil. Los datos fueron procesados por la estadística descriptiva. Los resultados evidencian que en la actualidad, la auditoria de enfermería está enfocada en los aspectos burocráticos, como contabilidad y finanzas, satisfaciendo las exigencias de las instituciones de salud. Cuando esa tendencia se proyecta al futuro, se observa una tendencia de cambio, incorporando la evaluación de la calidad de la atención en salud. En relación a los métodos actuales, los profesionales actúan según la forma retrospectiva, interna, ocupándose de las etapas de recolección y análisis de los pagos de cuentas. A medida que la auditoria de enfermería incorporar la evaluación de la calidad en su práctica, según la tendencia para los próximos cinco años, los métodos para su realización se modificarán, agregando diferentes maneras para sostener la nueva realidad.

DESCRIPTORES: Auditoría de enfermería. Economia. Calidad de la atención de salud. 


\section{INTRODUÇAO}

A reforma do setor saúde implementada nos anos 1990 tem implicado em alterações nos serviços de saúde, particularmente no que tange aos aspectos ideológicos e econômicos, na forma de produção desses serviços.

A idéia fundamental é administrar organizações, em especial hospitais e clínicas especializadas, com garantia de uma relação custo/benefício adequada. Nesta perspectiva as operadoras de seguros conquistam espaços na atenção à saúde e recrutam enfermeiros para gerenciar segundo essa lógica mercadológica.

No entanto, há de se considerar que o cliente nas instituições de saúde, cujos negócios são a prestação de serviços, deve sempre ser o centro da atenção. Neste contexto, para se manterem no mercado, os profissionais e as instituições necessitarão desenvolver uma nova filosofia de trabalho, norteada por questões financeiras e no atendimento de qualidade, permitindo assim, um crescimento de forma sustentada, onde cada passo a ser dado pautado nesta filosofia, estará engajado em bases sólidas.

Com as transformações mundiais, mudanças de paradigmas, exigências profissionais, principalmente, com relação à aspectos éticos e atitudinais, novos contextos permeiam a agenda dos gestores, determinando tendências às quais espera-se que os profissionais atendam. ${ }^{1}$

A tendência da função do enfermeiro auditor, em torno de uma concepção mesclada de controle de custo e de melhoria da qualidade, pode resultar de um panorama de reestruturação da produção em saúde demarcada por uma nova lógica de gestão das organizações, denominada Atenção Gerenciada.

O modelo de Atenção Gerenciada constituise em uma prática de gestão que evidencia a necessidade de gerenciar os cuidados de saúde, possibilitando um equacionamento entre racionalização dos custos de produção das intervenções e qualidade dos serviços prestados, tendo como objetivo criar uma capacidade competitiva. ${ }^{2}$

Observa-se que nos hospitais não há consenso quanto à ênfase na assistência focada nas necessidades humanas, em detrimento dos custos. Em alguns momentos, prevalece a assistência independente dos custos e, em outros a realização da assistência com o mínimo de recursos. ${ }^{3}$

$\mathrm{O}$ auditor em enfermagem, em um processo de educação continuada e articulação com a equipe assistencial e administrativa, pode intermediar a discussão desses paradigmas na instituição. Nesse contexto, justifica-se o desenvolvimento deste estudo.

\section{OBJETIVO}

Identificar tendências atuais e futuras (próximos cinco anos) da função do enfermeiro auditor no mercado de trabalho em saúde.

\section{METODOLOGIA}

Foi realizado um estudo exploratório de natureza quantitativa, através da mensuração de opinião utilizando a técnica Delphi.

Com base nas referências conceituais e metodológicas sobre auditoria de enfermagem, encontradas na literatura científica selecionada sobre o tema, procedeu-se à elaboração do instrumento conforme orientação da técnica Delphi.

O instrumento foi elaborado abrangendo a caracterização dos participantes e dados de opinião, subdivididos em item I composto por questões de tendências da prática da auditoria de enfermagem e a previsão do seu desenvolvimento nos próximos cinco anos e, item II composto por questões que objetivaram elencar prioridades a serem implementadas para o desenvolvimento da prática da auditoria de enfermagem.

O instrumento foi submetido preliminarmente a dois juizes, enfermeiros auditores para apreciação, tendo em vista a análise da clareza do texto das informações contidas, recebendo parecer favorável.

A técnica Delphi, é uma forma de abordagem que permite obter consenso por meio da opinião de um grupo de especialistas sobre uma matéria de interesse; ${ }^{4}$ busca deduzir, refinar e gerar uma opinião final a partir de um grupo de especialistas (denominado juízes ou painelistas), sendo também usada para estimar parâmetros desconhecidos.

Trata-se de um questionário, que circula diversas vezes (denominado rodadas), pelo grupo de especialistas, preservando o anonimato das respostas. Na primeira rodada os especialistas recebem um questionário, quando são solicitados a responder com respostas quantitativas apoiadas por justificativas e informações qualitativas, sendo realizado feedback de respostas do grupo para as rodadas subsequentes. ${ }^{5}$

O primeiro passo para a implementação da técnica foi de identificar os especialistas sobre a temática estudada, compondo assim o grupo de 
juizes ou painelistas para opinar sobre as tendências da auditoria de enfermagem.

Foram critérios de inclusão dos participantes do estudo: docentes da área de administração em enfermagem de universidades públicas e privadas brasileiras, enfermeiros auditores de instituições públicas, privadas e operadoras de saúde e enfermeiros em cargos de gerência. A seleção foi realizada por meio de contatos do pesquisador pela internet, por meio de sites de instituições de saúde brasileiras que disponibilizaram os endereços eletrônicos destes enfermeiros, sendo selecionado o total de 198 enfermeiros. Após essa consulta 78 responderam positivamente a intenção de colaborar com a pesquisa. A coleta de dados foi realizada no período de agosto a outubro de 2006.

Ressalta-se que o contexto do que está ocorrendo na atualidade refere-se ao período em que foi realizada a coleta de dados e, o futuro, refere-se aos cinco anos subsequentes a este período.

Atendendo opção dos participantes, o instrumento de pesquisa e o Termo de Consentimento Informado e Esclarecido, foram enviados via correio eletrônico para 61 participantes e, por meio de cartas com envelopes pré-selados anexos para 17 participantes. Foram realizadas duas rodadas sendo que, na primeira 54 participantes responderam o instrumento e, na segunda, 34 instrumentos foram respondidos. O consenso foi alcançado nos itens que obtiveram $70 \%$ ou mais de concordância. Após a primeira rodada o instrumento foi reformulado, sendo retirados cinco subitens que obtiveram consenso.

Os dados foram codificados manualmente, tratados segundo cálculo de porcentagem e digitados em uma base de dados do programa Microsoft Office Excel. Portanto, os resultados estão apresentados mediante estatística descritiva, considerando o objetivo da pesquisa, e a matriz de quesitos que compôs o instrumento de coleta de dados.

O painel final dos participantes constituiu-se de $76,5 \%$ enfermeiros com sete anos ou mais de tempo de formação, sendo que, $41,2 \%$ atuavam na docência, 52,9\% em serviços de saúde e 5,9\% atuavam nas duas áreas; a maioria dos participantes $(55,9 \%)$ atuavam no estado de São Paulo. Quanto à experiência, na área de administração em enfermagem, correspondeu a $88,2 \%$ dos participantes e quanto à experiência em auditoria de enfermagem 64,7\%; com relação a pós graduação, $55,9 \%$ possuiam em administração em enfermagem e 38,2\% em auditoria de enfermagem.

Esta pesquisa foi submetida à apreciação do Comitê de Ética em Pesquisa da Escola de Enfer- magem de Ribeirão Preto da Universidade de São Paulo, obtendo aprovação para sua realização sob o N $\mathrm{N}^{\mathrm{0}} 0630 / 2005$.

\section{RESULTADOS E DISCUSSÃO}

Para análise consideramos o contexto do sistema de saúde brasileiro e a conformação do papel do enfermeiro nas organizações de saúde. Compreende-se o sistema de serviços de saúde no Brasil como um modelo segmentado, ou seja, organiza-se agregando diferentes clientelas, em segmentos institucionais singulares, onde cada segmento, público ou privado, exercita as funções de financiamento, regulação e prestação de serviços para sua clientela particular. ${ }^{6}$

Desta forma, a atenção à saúde é ofertada por três sistemas: o Sistema Único de Saúde (SUS) que se constitui no sistema público estruturado por órgãos e instituições públicas federais, estaduais e municipais, da administração direta e indireta; o Sistema de Atenção Médica Supletiva (SAMS), o qual incorpora as modalidades assistenciais de medicina de grupo, a autogestão, a cooperativa médica e o segurosaúde e, por último, o Sistema de Desembolso Direto (SDD) que trata de uma prática liberal que prevê pagamento direto, pelo cidadão, de seus gastos com saúde.

Diante desse panorama do setor saúde, buscamos, na literatura, parâmetros acerca da função do enfermeiro auditor e apreendemos tratar-se de um processo de exame, verificação, interpretação e valorização crítica de situações frente as normas de eficácia, eficiência, qualidade e produtividade, bem como da identificação das capacidades da organização, da emissão de opinião sobre aspectos intangíveis (relações e conflitos) que afetam o desenvolvimento, emitindo recomendações para garantir integridade patrimonial, organizacional, informativa e a gestão. ${ }^{7}$

Nossa pesquisa identificou dois eixos de análise que conformam as tendências da função do enfermeiro auditor, os quais apresentamos na sequência.

\section{O enfermeiro auditor e seu método de tra- balho}

O método de trabalho do enfermeiro auditor decorre de um ideário profissional, o qual está articulado com um campo de conhecimentos e práticas de gestão, que se diferenciam segundo 
lógicas organizacionais de natureza pública ou privada. As práticas e os métodos de auditoria de enfermagem desenvolvem-se mais amplamente em instituições privadas regidas pelo modelo de Atenção Gerenciada, o que tem gerado questionamentos na comunidade de enfermeiros, a fim de evitar que a função do auditor se encaminhe para um controle administrativo que se sobreponha às ações de saúde com a pretensão de reduzir tratamentos de alto custo. A Tabela 1 apresenta os resultados encontrados acerca dos métodos de auditoria de enfermagem.

Tabela 1 - Ação de auditar na atualidade e no futuro, segundo enfermeiros experts em auditoria, segunda rodada de opiniões. Brasil, 2006

\begin{tabular}{|c|c|c|c|c|c|c|c|c|}
\hline \multirow{3}{*}{ Etapas da ação de auditar } & \multicolumn{4}{|c|}{$\begin{array}{c}\text { Atualidade } \\
\mathrm{n}=34\end{array}$} & \multicolumn{4}{|c|}{$\begin{array}{c}\text { Futuro } \\
\mathrm{n}=34\end{array}$} \\
\hline & \multicolumn{2}{|c|}{$\begin{array}{l}\text { Pouco } \\
\text { provável }\end{array}$} & \multicolumn{2}{|c|}{$\begin{array}{l}\text { Muito } \\
\text { provável }\end{array}$} & \multicolumn{2}{|c|}{$\begin{array}{l}\text { Pouco } \\
\text { provável }\end{array}$} & \multicolumn{2}{|c|}{$\begin{array}{l}\text { Muito } \\
\text { provável }\end{array}$} \\
\hline & $f$ & $\%$ & $f$ & $\%$ & $f$ & $\%$ & $f$ & $\%$ \\
\hline $\begin{array}{l}\text { Envolve objetivos, coleta e análise de dados da } \\
\text { assistência de enfermagem durante a internação } \\
\text { até a alta e relatório técnico }\end{array}$ & 27 & 79,4 & 7 & 20,6 & 9 & 26,5 & 25 & 73,5 \\
\hline $\begin{array}{l}\text { Análise da estrutura, processo e resultado para } \\
\text { avaliação integrada }\end{array}$ & 30 & 88,2 & 4 & 11,8 & 9 & 26,5 & 25 & 73,5 \\
\hline $\begin{array}{l}\text { Coleta de dados da enfermagem e análise de pa- } \\
\text { gamento com objetivo de impor ou diminuir glosas }\end{array}$ & 8 & 23,5 & 26 & 76,5 & 5 & 14,7 & 29 & 85,3 \\
\hline
\end{tabular}

O uso do método de auditoria de enfermagem envolvendo as etapas de definição de objetivos, coleta de dados sobre todos os processos da assistência de enfermagem envolvidos desde a admissão até a alta do paciente e análise dos dados com elaboração do relatório técnico, obteve consenso como pouco provável de ser praticado na atualidade $(79,4 \%)$ e muito provável que ocorra nos próximos cinco anos (73,5\%).

A realização da análise da estrutura, processo e resultado da assistência de enfermagem, tendo em vista uma avaliação integrada e ampliada, como etapas do método da auditoria em enfermagem, obteve consenso como pouco provável que esteja sendo utilizada na atualidade $(88,2 \%)$ e muito provável que venha a ser utilizada nos próximos cinco anos (73,5\%).

A conformação do método da auditoria de enfermagem contemplando a coleta de dados e análise dos pagamentos das contas hospitalares, relativos à sub área de enfermagem a fim de impor glosas ou diminuí-las, foi considerada pelos participantes como muito provável na atualidade $(76,5 \%)$ e nos próximos cinco anos $(85,3 \%)$.

Embora as etapas apontadas como método utilizado na atualidade estejam configuradas conforme o perfil exigido pelas instituições de saúde, focado na área contábil e financeira; na área da saúde, o processo para a realização da auditoria tem sido apontado como prática adequada sua execução com cinco etapas básicas: planificação dos objetivos, delineamento das atividades abrangendo a previsão de recursos necessários e áreas envolvidas, análise e avaliação da informação, apresentação e divulgação dos resultados e adoção de ações para melhoria do serviço. ${ }^{8}$

Ainda com relação ao método da auditoria de enfermagem, a procedência dos dados/informações para a realização está detalhada na Tabela 2.

A realização de consulta dos dados procedentes dos registros de enfermagem do prontuário do paciente, dos manuais de procedimentos e rotinas de enfermagem e dos manuais de padrões de assistência de enfermagem, na auditoria de enfermagem, foi considerada como muito provável na atualidade (73,5\%) e nos próximos cinco anos (85,3\%).

A coleta de dados para a realização da auditoria em enfermagem baseando-se no prontuário do paciente e dos documentos relativos à conta hospitalar, foi considerada como consenso na atualidade $(88,2 \%)$ e nos próximos cinco anos $(94,1 \%)$.

A utilização dos prontuários na realização da auditoria possibilita identificar problemas e orientar a equipe e a instituição quanto ao registro apropriado das ações, bem como o respaldo ético e legal, permite ainda apontar desvios, propiciar propostas e estratégias para melhoria da qualidade da assistência. ${ }^{9}$ 
Tabela 2 - Procedência dos dados/informações para auditoria de enfermagem na atualidade e no futuro, segundo enfermeiros experts em auditoria, segunda rodada de opiniões. Brasil, 2006

\begin{tabular}{|c|c|c|c|c|c|c|c|c|c|c|c|c|}
\hline \multirow{3}{*}{ Procedência dos dados/informações } & \multicolumn{6}{|c|}{$\begin{array}{c}\text { Atualidade } \\
\mathrm{n}=34\end{array}$} & \multicolumn{6}{|c|}{$\begin{array}{c}\text { Futuro } \\
\mathrm{n}=34\end{array}$} \\
\hline & \multicolumn{2}{|c|}{$\begin{array}{l}\text { Pouco } \\
\text { provável }\end{array}$} & \multicolumn{2}{|c|}{$\begin{array}{l}\text { Muito } \\
\text { provável }\end{array}$} & \multicolumn{2}{|c|}{$\begin{array}{l}\text { Sem } \\
\text { resposta }\end{array}$} & \multicolumn{2}{|c|}{$\begin{array}{l}\text { Pouco } \\
\text { provável }\end{array}$} & \multicolumn{2}{|c|}{$\begin{array}{l}\text { Muito } \\
\text { provável }\end{array}$} & \multicolumn{2}{|c|}{$\begin{array}{l}\text { Sem } \\
\text { resposta }\end{array}$} \\
\hline & $f$ & $\%$ & f & $\%$ & $f$ & $\%$ & $f$ & $\%$ & $f$ & $\%$ & $f$ & $\%$ \\
\hline $\begin{array}{l}\text { Registro de enfermagem no prontuário dos } \\
\text { pacientes, nos manuais de procedimentos, } \\
\text { rotinas e padrões de assistência }\end{array}$ & 9 & 26,5 & 25 & 73,5 & - & - & 5 & 14,7 & 29 & 85,3 & - & - \\
\hline $\begin{array}{l}\text { Prontuário do paciente e documentos refe- } \\
\text { rentes a conta hospitalar }\end{array}$ & 4 & 11,8 & 30 & 88,2 & - & - & 2 & 5,9 & 32 & 94,1 & - & - \\
\hline $\begin{array}{l}\text { Observação direta da assistência, entrevis- } \\
\text { tas com pacientes, familiares e profissionais } \\
\text { acerca da estrutura, processo e resultado }\end{array}$ & 29 & 85,3 & 4 & 11,8 & 1 & 2,9 & 12 & 35,3 & 22 & 64,7 & - & - \\
\hline
\end{tabular}

Em relação à utilização dos dados coletados para a auditoria de enfermagem serem procedentes da observação direta à assistência ao paciente, de entrevistas com o mesmo, familiares e equipe de enfermagem e de saúde acerca da estrutura, processos e resultados da assistência de enfermagem, $85,3 \%$ dos participantes consideraram pouco provável serem estas as fontes de informações consultadas na atualidade. No futuro esta perspectiva não obteve consenso entre os participantes.

Embora esse quesito obteve consenso como muito provável que não esteja ocorrendo na atualidade e sem consenso quando projetado para o futuro, ressalta-se que o uso de questionários, entrevistas e observações já fazem parte da realidade dos métodos de processo de auditoria nos programas de avaliação externa de alguns hospitais ou serviços de saúde, que os aplicam de forma participativa abrangendo os vários agentes da assistência. ${ }^{10}$

Desse modo, o método para realização da auditoria de enfermagem, de acordo com a opinião dos participantes, está sendo aplicado na atualidade da seguinte forma:

- contemplando as etapas de coleta de dados e de análise dos pagamentos das contas hospitalares, relativos à sub área de enfermagem, a fim de impor glosas ou diminuí-las;

- coletando os dados dos prontuários dos pacientes, registros de enfermagem, manuais de procedimentos, e rotinas e manuais de padrões da assistência;

- coletando os dados também do prontuário do paciente e documentos relativos à conta hospitalar.
Os participantes da pesquisa consideraram que o método da auditoria de enfermagem, no futuro (próximos cinco anos), será da seguinte forma:

- de maneira retrospectiva, utilizando as informações do prontuário do paciente e do sistema gerencial da assistência de enfermagem e também de maneira concorrente, com acompanhamento dos processos da assistência de enfermagem no local da internação;

- envolverá as etapas: definição de objetivos, coleta de dados acerca dos processos da assistência de enfermagem, da admissão até a alta do paciente e análise dos dados com elaboração do relatório técnico;

- contemplará as etapas: análise da estrutura, do processo e do resultado da assistência de enfermagem, considerando visão integrada e ampliada;

- contemplará as etapas: de coleta dos dados e análise dos pagamentos das contas hospitalares relativas à sub área de enfermagem, a fim de impor glosas ou diminuí-las;

- coleta dos dados procedentes dos prontuários dos pacientes, em especial, dos registros de enfermagem, dos manuais de procedimentos e rotinas e de padrões da assistência de enfermagem e coleta dos dados coletados procedentes do prontuário do paciente e de documentos relativos à conta hospitalar.

Para os próximos cinco anos, o item procedência dos dados através da observação direta da assistência, entrevistas com pacientes, familiares e profissionais quanto a estrutura, processo e resultado, também não foi alcançado o consenso entre os painelistas. 


\section{A função do enfermeiro auditor e suas fina- lidades}

$\mathrm{O}$ ato de auditar em enfermagem cumpre uma finalidade institucional que pode estar circunscrita ao serviço de enfermagem ou ampliar sua abrangência, alcançando motivações da direção de hospitais e serviços de saúde em geral. Apresenta-se, na tabela 3, os dados encontrados referentes ao quesito finalidade da função do enfermeiro auditor.

Tabela 3 - Finalidade da função do enfermeiro auditor na atualidade e no futuro, segundo enfermeiros experts em auditoria, segunda rodada de opiniões. Brasil, 2006

\begin{tabular}{|c|c|c|c|c|c|c|c|c|c|c|c|c|}
\hline \multirow{3}{*}{ Finalidades da auditoria } & \multicolumn{6}{|c|}{$\begin{array}{c}\text { Atualidade } \\
n=34\end{array}$} & \multicolumn{6}{|c|}{$\begin{array}{c}\text { Futuro } \\
n=34\end{array}$} \\
\hline & \multicolumn{2}{|c|}{$\begin{array}{l}\text { Pouco } \\
\text { provável }\end{array}$} & \multicolumn{2}{|c|}{$\begin{array}{c}\text { Muito } \\
\text { provável }\end{array}$} & \multicolumn{2}{|c|}{$\begin{array}{c}\text { Sem } \\
\text { resposta }\end{array}$} & \multicolumn{2}{|c|}{$\begin{array}{l}\text { Pouco } \\
\text { provável }\end{array}$} & \multicolumn{2}{|c|}{$\begin{array}{c}\text { Muito } \\
\text { provável }\end{array}$} & \multicolumn{2}{|c|}{$\begin{array}{l}\text { Sem } \\
\text { resposta }\end{array}$} \\
\hline & $f$ & $\%$ & $f$ & $\%$ & $f$ & $\%$ & $f$ & $\%$ & $f$ & $\%$ & $f$ & $\%$ \\
\hline $\begin{array}{l}\text { Contribuir para o serviço de enfermagem } \\
\text { e a direção do hospital delinearem ações } \\
\text { corretivas }\end{array}$ & 23 & 67,6 & 11 & 32,4 & - & - & 6 & 17,6 & 28 & 82,4 & - & - \\
\hline $\begin{array}{l}\text { Contribuir para reformulação de práticas } \\
\text { inadequadas de enfermagem e indicação } \\
\text { de educação em serviço }\end{array}$ & 23 & 67,7 & 10 & 29,4 & 1 & 2,9 & 7 & 20,6 & 27 & 79,4 & - & - \\
\hline $\begin{array}{l}\text { Contribuir para negociação entre dirigen- } \\
\text { tes do hospital e convênios de saúde para } \\
\text { melhoria da qualidade da assistência de } \\
\text { enfermagem }\end{array}$ & 24 & 70,6 & 10 & 29,4 & - & - & 13 & 38,2 & 21 & 61,8 & - & - \\
\hline $\begin{array}{l}\text { Contribuir para instalar negociação entre } \\
\text { dirigentes do hospital e convênios de saúde } \\
\text { para comprovar pagamentos, questionar e } \\
\text { rever glosas }\end{array}$ & 8 & 23,5 & 26 & 76,5 & - & - & 3 & 8,8 & 31 & 91,2 & - & - \\
\hline
\end{tabular}

O delineamento de ações corretivas pela gerência do serviço de enfermagem e pela direção do hospital foi considerado por $82,4 \%$ como finalidade que, muito provavelmente, será praticada nos próximos cinco anos. No entanto, este tópico não obteve consenso entre os participantes quando refletido na prática da atualidade.

A reformulação de práticas inadequadas, indicando processos de educação em serviço, não obteve consenso entre os participantes na atualidade e obteve, com 79,4\% de concordância, como sendo uma etapa que, muito provavelmente, será praticada nos próximos cinco anos.

A realização de negociação entre representantes do hospital e do convênio de saúde para questionar e melhorar a qualidade da assistência de enfermagem foi considerado por $70,6 \%$ dos participantes como sendo pouco provável na atualidade. No entanto, quando esta possibilidade é projetada para os próximos cinco anos, não há consenso entre os painelistas.

Instalar negociação entre os representantes do hospital e do convênio de saúde para compro- var pagamentos de contas relativas à assistência de enfermagem e rever glosas apontadas foi considerada como muito provável por $76,5 \%$ dos painelistas na perspectiva atual; $91,2 \%$ consideraram muito provável que a auditoria em enfermagem tenha esta finalidade nos próximos cinco anos.

Assim, a finalidade da auditoria de enfermagem na atualidade, segundo as opiniões dos participantes da pesquisa, está restrita à comprovação de pagamento de contas hospitalares relativa à assistência de enfermagem, questionando e revendo glosas apontadas, realizando negociações entre os representantes do hospital e do convênio.

Nos próximos cinco anos, os participantes consideraram que a finalidade da auditoria de enfermagem será apontar inadequações na assistência de enfermagem, reformulando suas práticas, indicando processos de educação em serviço, delineando ações corretivas pela gerência do serviço de enfermagem e direção do hospital. Consideram também como finalidade a comprovação de pagamentos de contas relativas à assistência de enfermagem, questionando e revendo glosas 
apontadas pelos representantes do hospital e do convênio de saúde.

Desse modo, vimos que no futuro há uma perspectiva de mudança de paradigma, com preocupações acerca da qualidade do serviço prestado. Iniciativas no sentido de garantir a assistência de qualidade estão cada vez mais emergentes no cenário atual, seja por movimento governamental, ou por entidades independentes, seja por pressão social ou pelos clientes corporativos que financiam o seguro saúde, que almejam retornos concretos em face ao investimento no serviço. ${ }^{11}$

Com relação à finalidade da auditoria de enfermagem na perspectiva atual não foram alcançados consensos entre os participantes nos itens: contribuição para o delineamento de ações corretivas pelo serviço de enfermagem e direção do hospital; contribuição para a reformulação de práticas de enfermagem inadequadas com indicação de educação em serviço. Na perspectiva futura não obteve consenso no item contribuição para instalar negociação entre dirigentes do hospital e convênios para melhoria da qualidade assistencial.

\section{CONSIDERAÇÕES FINAIS}

No Brasil a partir da década de 20, do século passado, com a criação dos primeiros Cursos de Enfermagem, as enfermeiras passaram a assumir posições administrativas no contexto hospitalar. ${ }^{12}$ Esse papel se fortalece ao longo dos anos com a forte atuação do enfermeiro em atividades relacionadas à área administrativa burocrática. ${ }^{13}$ Pode-se dizer que esse cenário ainda não foi alterado, muito embora, neste princípio de século, a preocupação com o cuidado de enfermagem e com a gerência desse cuidado tem conduzido a um repensar sobre o papel do enfermeiro na sociedade atual, tendo em vista a garantia da qualidade da assistência de enfermagem prestada aos cidadãos.

No entanto, ao analisar os resultados dessa investigação, os enfermeiros experts na temática da auditoria de enfermagem apontam que as organizações de saúde e os enfermeiros auditores fortalecem o estilo de gerência burocrática, tendo em vista o controle de custos. Assim a tendência da função do enfermeiro auditor na atualidade, retrata sua dimensão burocrática, de cunho contábil e financeiro, contemplando as atuais exigências das instituições de saúde. Quando essa tendência é reportada para o futuro, observa-se uma projeção de mudança conceitual sendo que os enfermeiros consideram que a auditoria de enfermagem in- corporará a avaliação da qualidade da assistência prestada ao paciente.

A exigência por parte das instituições de saúde, da atuação dos enfermeiros e de outros profissionais da saúde na área contábil e financeira, tem o intuito de adequar os custos e otimizar recursos mediante enfoque empresarial e mercadológico. Pode-se dizer que esse mesmo enfoque começa a aglutinar uma perspectiva de qualidade assim já se percebe, nas opiniões dos participantes da pesquisa, um movimento quanto ao posicionamento favorável à mudança de paradigma, com a incorporação da avaliação da qualidade do serviço prestado.

Quando a assistência ao paciente é de má qualidade, os custos da internação aumentam, portanto, cria-se a necessidade de habilitação do enfermeiro auditor para realizar a auditoria agregando a vertente contábil e de qualidade. Essa é uma forte tendência a ser implementada nos próximos anos, segundo a opinião dos participantes, uma vez que instituições de saúde com custos otimizados, passam a ter subsídios financeiros para investimentos em sua estrutura (recursos humanos, tecnológicos e físicos), oferecendo, assim, suporte para as ações de melhoria da qualidade. ${ }^{14}$

Embora os resultados da pesquisa apontem o foco na atualidade apenas voltado a área contábil e financeira, já nota-se na prática, um movimento voltado para a qualidade, inclusive por parte das operadoras de saúde, as quais, por meio da realização de auditorias, avaliam a qualidade da assistência prestada nas instituições por elas contratadas para revalidar contratos, adequar tabelas de preços e avaliar se o nível da assistência está de acordo com seus princípios.

Com relação aos métodos para a realização da auditoria de enfermagem, a tendência é a de proceder segundo a forma retrospectiva, utilizando-se de dados coletados do prontuário do paciente, relativos à conta hospitalar, manuais de procedimentos, rotinas e padrões da assistência, sendo realizados por enfermeiros da própria instituição (tipo interna), contemplando as etapas de coleta e análise dos pagamentos da conta. Tais métodos estão intimamente relacionados à concepção de auditoria de enfermagem identificada na perspectiva atual.

Sendo assim, segundo a opinião dos participantes dessa pesquisa, o método para realização da auditoria não está sendo feito de forma abrangente segundo as etapas apontadas pela literatura e nem tão pouco com uma avaliação integrada, cenário este que se reverterá segundo tendência para o futuro. 
A medida que a auditoria de enfermagem incorporar à sua prática a avaliação da qualidade da assistência prestada ao paciente, conforme tendência apontada para os próximos cinco anos, os métodos para sua realização se modificarão, agregando diferentes maneiras para sustentar a nova realidade. Ressaltamos que a escolha do método a ser utilizado para a realização da auditoria de enfermagem deverá estar pautada no objetivo da avaliação a que se destina e, nessa perspectiva a função do enfermeiro auditor poderá ser enriquecida e guiada não somente pelas necessidades financeiras da instituição mas, principalmente, pelas necessidades de saúde dos clientes.

Em relação a procedência dos dados, foi identificado na opinião dos participantes, que atualmente não é contemplada a realização de observações diretas, nem tão pouco o envolvimento dos agentes participantes da assistência no processo de realização da auditoria. Quando o cenário é projetado para os próximos cinco anos, não houve consenso entre os participantes quanto a inserção destes no processo.

É válido ressaltar que a participação dos agentes envolvidos na assistência ao paciente é de suma importância, sendo enriquecedor para consolidar o processo da realização da auditoria e tem sido valorizado na atualidade nas auditorias realizadas em programas de qualidade.

Evidencia-se que a perspectiva futura de mudança na configuração atual da auditoria de enfermagem se deve, principalmente, às exigências do mercado em saúde que busca desenvolver uma lógica de gestão de qualidade. Para que a auditoria de enfermagem possa cumprir sua função é de suma importância a utilização de métodos e, estes não devem se distanciar do fio condutor da profissão que é o cuidado de enfermagem prestado ao paciente,

\section{REFERÊNCIAS}

1. Cunha ICKO, Francisco Neto RGX. Competências gerenciais de enfermeiras: um novo desafio? Texto Contexto Enferm. 2006 Jul-Set; 15(3):479-82.
2. Merhy EE. Reflexões sobre as tecnologias não materiais em saúde e a reestruturação produtiva do setor: um estudo sobre a micropolítica do trabalho vivo [tese]. Campinas (SP): Universidade de Campinas, Faculdade de Ciências Médicas; 2000.

3. Burmester H. Reflexiones sobre los programas hospitalarios de garantía de calidad. Rev Panam Salud Publica. 1997 Feb;1(2):149-54.

4. Faro ACM. Técnica delphi na validação das intervenções de enfermagem. Rev Esc Enferm USP. 1997 Ago-Dez; 31(2):259-70.

5. Wright JTC, Giovinazzo RA. Delphi: uma ferramenta de apoio ao planejamento prospectivo. Cad Pesqui Admin. 2000 Abr-Jun; 1(12):54-65.

6. Mendes EV. Os grandes dilemas do SUS. Salvador (BA): Casa da Qualidade Editora; 2001.

7. Consiall I, Pueyo D. A auditoría interna em hospitales. Gestión em Salud. 2003 Abr; 2(5):44-55.

8. Melo MB, Vaitsman J. Auditoria e avaliação no Sistema Único de Saúde. São Paulo em Perspectiva. 2008 Jan-Jun; 22(1):152-64.

9. Setz VG, D’Innocenzo M. Avaliação da qualidade dos registros de enfermagem no prontuário por meio da auditoria. Acta Paul Enferm. 2009 Jun; 22(3):313-17.

10. Novaes HMD. Avaliação de programas, serviços e tecnologias em saúde. Rev. Saúde Pública. 2000 Out; 34(5):547-49.

11. Escrivão Junior A, Koyama MF. O relacionamento entre hospitais e operadoras de planos de saúde no âmbito do Programa de Qualificação da Saúde Suplementar da ANS. Ciência Saúde Coletiva. 2007 Jul-Ago; 12(4):903-14.

12. Ferraz CA. A transfiguração da administração em enfermagem: da gerência científica à gerência sensível [tese]. Ribeirão Preto (SP): Universidade de São Paulo, Escola de Enfermagem de Ribeirão Preto; 1995.

13. Trevizan MA. Enfermagem hospitalar: administração e burocracia. Brasília (DF): Universidade de Brasília; 1988.

14. Goto DYN. Instrumento de auditoria técnica de conta hospitalar: mensurando perdas e avaliando a qualidade da assistência. Curitiba (PR): Universidade Federal do Paraná; 2001. 